\title{
Rastreamento de transtorno depressivo e/ou ansioso em discentes do curso de farmácia de uma universidade pública brasileira
}

\author{
Screening for depressive and/or anxiety disorder in pharmacy course students at a Brazilian public \\ university
}

Cribado de trastorno depresivo y / o de ansiedad en estudiantes del curso de farmacia de una universidad pública brasileña

Recebido: 15/09/2021 | Revisado: 19/09/2021 | Aceito: 20/09/2021 | Publicado: 22/09/2021

\author{
Maria Thaynam de Lima Carvalho \\ ORCID: https://orcid.org/0000-0001-5011-3677 \\ Universidade Federal da Paraíba, Brasil \\ E-mail: thaynanlima94@ hotmail.com \\ Camila Maria Cordeiro Dias \\ ORCID: https://orcid.org/ 0000-0002-6173-5145 \\ Universidade Federal do Rio Grande do Norte, Brasil \\ E-mail: camilamcordeirodias@gmail.com \\ Rafael Marinho de Lima Paiva \\ ORCID: https://orcid.org/0000-0001-5389-9310 \\ Universidade Federal da Paraíba, Brasil \\ E-mail: rafaelmarinholtf@gmail.com \\ Vinícius Soares Ribeiro \\ ORCID: https://orcid.org/ 0000-0003-0715-0557 \\ Universidade Federal da Paraíba, Brasil \\ E-mail: vinicius.rbxz@gmail.com \\ Cleyton Oliveira Bezerra \\ ORCID: https://orcid.org/0000-0002-1564-5704 \\ Universidade Federal da Paraíba, Brasil \\ E-mail: cobmme3@gmail.com \\ Cinthia Caldas Rios \\ ORCID: https://orcid.org/0000-0001-5596-0007 \\ Universidade Federal da Paraíba, Brasil \\ E-mail: cinthia.crios@gmail.com \\ Thais Teles de Souza \\ ORCID: https://orcid.org/0000-0002-6820-4259 \\ Universidade Federal da Paraíba, Brasil \\ E-mail: thaisteles.ufpb@gmail.com \\ Walleri Christini Torelli Reis \\ ORCID: https://orcid.org/0000-0001-6911-4792 \\ Universidade Federal da Paraíba, Brasil \\ E-mail: wallerictr@gmail.com
}

\begin{abstract}
Resumo
O objetivo do estudo foi estimar a prevalência de rastreamento positivo para transtorno depressivo e ansioso, em discentes do curso de graduação em Farmácia, de uma universidade pública, no estado da Paraíba. Questionários validados para rastreamento de depressão (PHQ9) e ansiedade (BAI) foram aplicados em estudantes de graduação em farmácia matriculados na universidade. Participaram da pesquisa alunos maiores de 18 anos, sem diagnóstico prévio de transtornos mentais. A pesquisa foi submetida ao Comitê de Ética em Pesquisas do Centro de Ciências da Saúde da Universidade Federal da Paraíba, sendo aprovada sob o Parecer no CAAE 15399019.4.0000.5188. Os resultados apontaram que $34 \%$ dos alunos avaliados apresentaram scores compatíveis com depressão moderada a grave e $18 \%$ com scores compatíveis com ansiedade moderada a grave. Foi observada correlação de Pearson positiva $(r=0,672)$ em relação aos scores do questionário dos transtornos depressivos e ansiosos. Foi identificada correlação positiva entre a idade e os scores do PHQ9 ( $\mathrm{r}=0,059)$. Não foi observada correlação entre idade e os scores do BAI, assim como entre o score da escala de PHQ-9 ( $\mathrm{r}=0,003)$ ou BAI $(r=0,033)$ em correlação com a renda dos acadêmicos. Os dados demonstram que existe uma elevada prevalência de possíveis transtornos mentais em alunos de farmácia da UFPB, sendo fundamental o cuidado e intervenção do farmacêutico para o acompanhamento e tratamento adequado, tendo em vista promover a melhoria da qualidade de vida dos estudantes universitários.
\end{abstract}

Palavras-chave: Rastreamento em saúde; Transtornos mentais; Estudantes de saúde. 


\begin{abstract}
The study aimed to estimate the prevalence of positive screening for depressive and anxiety disorders in undergraduate pharmacy students at a public university in the state of Paraíba. Validated questionnaires for screening for depression (PHQ9) and anxiety (BAI) were administered to undergraduate pharmacy students enrolled at the university. Students over 18 years old, with no previous diagnosis of mental disorders, participated in the research. The research was submitted to the Research Ethics Committee of the Health Sciences Center of the Federal University of Paraíba, being approved under Opinion No. CAAE 15399019.4.0000.5188. The results showed that $34 \%$ of the evaluated students had scores compatible with moderate to severe depression and $18 \%$ with scores compatible with moderate to severe anxiety. A positive Pearson correlation $(r=0.672)$ was observed about the scores of the questionnaire for depressive and anxiety disorders. A positive correlation was identified between age and PHQ9 scores $(r=0.059)$. There was no correlation between age and BAI scores, as well as between the PHQ-9 ( $r=0.003)$ or BAI ( $r=0.033)$ scale score in correlation with the students' income. The data show that there is a high prevalence of possible mental disorders in pharmacy students at UFPB, with the care and intervention of the pharmacist being essential for proper monitoring and treatment, to promote an improvement in the quality of life of university students.
\end{abstract}

Keywords: Health screening; Mental disorders; Health students.

\title{
Resumen
}

El objetivo del estudio fue estimar la prevalencia de cribado positivo para trastornos depresivos y de ansiedad en estudiantes de pregrado de farmacia de una universidad pública del estado de Paraíba. Se administraron cuestionarios validados para el cribado de depresión (PHQ9) y ansiedad (BAI) a estudiantes de farmacia de pregrado matriculados en la universidad. Participaron de la investigación estudiantes mayores de 18 años, sin diagnóstico previo de trastorno mental. La investigación fue sometida al Comité de Ética en Investigación del Centro de Ciencias de la Salud de la Universidad Federal de Paraíba, siendo aprobada bajo Dictamen No. CAAE 15399019.4.0000.5188. Los resultados mostraron que el 34\% de los estudiantes evaluados tenían puntajes compatibles con depresión moderada a severa y el $18 \%$ con puntajes compatibles con ansiedad moderada a severa. Se observó una correlación de Pearson positiva $(\mathrm{r}=0,672)$ en relación con las puntuaciones del cuestionario para trastornos depresivos y de ansiedad. Se identificó una correlación positiva entre la edad y las puntuaciones de PHQ9 $(r=0,059)$. No hubo correlación entre la edad y los puntajes BAI, así como entre el puntaje de la escala PHQ-9 $(r=0.003)$ o BAI $(r=0.033)$ en correlación con los ingresos de los estudiantes. Los datos muestran que existe una alta prevalencia de posibles trastornos mentales en los estudiantes de farmacia de la UFPB, y la atención e intervención del farmacéutico es fundamental para un adecuado seguimiento y tratamiento, con miras a promover la mejora de la calidad de vida de los estudiantes universitários.

Palabras clave: Cribado de Salud; Desordenes mentales; Estudiantes de salud.

\section{Introdução}

$\mathrm{O}$ ingresso na universidade representa um período de grande importância para os estudantes, visto que é a possibilidade de realização da formação profissional. Porém, a trajetória acadêmica inicialmente é marcada por uma árdua fase de adaptação, relacionada a mudanças no estilo de vida do indivíduo, novos relacionamentos interpessoais, distanciamento parental e a descoberta da autonomia e suas consequências(Chao \& Chu-Lien, 2012). Esse contexto está intimamente relacionado com elevados níveis de estresse, distúrbios no apetite, problemas de concentração e relacionados ao sono(Thurber et al., 2007). As dificuldades associadas à vida acadêmica não são restritas apenas ao período de adaptação às novas responsabilidades, elas podem perdurar ao longo da graduação, com a necessidade do desenvolvimento de competências que exigem maior responsabilidade, isso é particularmente evidente na área da saúde(Cestari et al., 2017; Oliveira, R. E. C; Morais, 2015).

Esse panorama pode predispor o desenvolvimento de transtornos mentais, como depressão e transtornos de ansiedade(Adewuya et al., 2006; Cavestro \& Rocha, 2006; Cerchiari et al., 2005; M.S. et al., 1991; Pillay et al., 2016). Apesar do adoecimento mental ser geralmente progressivo, o estigma e a negligência para procurar ajuda fazem com que os quadros se agravem e culminem muitas vezes em consequências dramáticas. Explicações possíveis para esse comportamento incluem: estigma social existente no uso de serviços psiquiátricos, a falta de tempo ou receio das consequências no próprio desempenho acadêmico(Vasconcelos et al., 2015). Muitos fatores podem ser preditores para um episódio depressivo, entre eles podemos citar aspectos neurovegetativos, motivacionais e/ou emotivos e socioeconômicos(Hasin et al., 2018). Desse modo, a depressão 
é uma doença que não deve ser negligenciada e precisa ser tratada adequadamente. Evidências apontam que a depressão é uma condição frequentemente não detectada na ausência de rastreamento, bem como as taxas de resposta ao tratamento são melhores se o tratamento for iniciado precocemente(O'connor, 2009; Pignone, M. P.; Gaynes, B. N.; Rushton, J. L.; Burchell, C. M, Orleans, C. T.; Mulrow, C. D.; lohr, 2002).

Dado isso, existem instrumentos de triagem validados internacionalmete, amplamente utilizados na atenção primaria, como o Patient Helth Questionnaire (PHQ-9), o qual possui fácil aplicação, boa sensibilidade e especificidade (Levis et al., 2019), utilizado para rastreio, estratificação e monitoramento da depressão (Kroenke et al., 2010). O rastreamento adequado, permite uma intervenção precoce e um desfecho mais promissor para o paciente (Gilbody et al., 2003; Pignone, M. P.; Gaynes, B. N.; Rushton, J. L.; Burchell, C. M, Orleans, C. T.; Mulrow, C. D.; Lohr, 2002). O rastreamento da ansiedade é tão importante quanto o rastreamento da depressão, e também sofre grande estigmatização social no âmbito da saúde mental, prejudicando a identificação e tratamento da condição (Wittchen \& Jacobi, 2005). Dessa maneira, também são utilizados instrumentos validados para o rastreamento e acompanhamento deste transtorno. Um dos mais utilizados na atualidade é o Beck Anxiety Inventory (BAI), que possui considerável confiabilidade(Magán et al., 2008; Osman et al., 2002; Vázquez Morejón et al., 2014).

O desempenho acadêmico de um estudante afetado por depressão ou ansiedade é prejudicado, sendo uma causa considerável para redução na sua qualidade de vida (McConville et al., 2017). É importante ressaltar que a interferência dessas doenças na vida acadêmica é evidenciada pela frequente associação à incapacidade de se concentrar, sendo observado nível de preocupação semelhante em estudantes ansiosos e depressivos, bem como estão relacionados com déficit na memória (Christopher \& MacDonald, 2005). É fundamental encontrar medidas preventivas eficazes para depressão e ansiedade no ambiente acadêmico, visto que elas geram custos significativos para a sociedade (Jadhakhan et al., 2019; Khushalani et al., 2018; Luppa et al., 2007). Desta forma, o presente estudo objetivou estimar a prevalência de rastreamento positivo para transtorno depressivo e ansioso em discentes do curso de graduação em Farmácia de uma universidade pública no estado da Paraíba, bem como identificar os sintomas mais prevalentes relacionados à depressão e ansiedade na população estudada.

\section{Metodologia}

O presente trabalho trata-se de um estudo observacional, transversal e de natureza quantitativa, realizado em estudantes de graduação em farmácia, vinculados ao Departamento de Ciências Farmacêuticas da Universidade Federal da Paraíba - UFPB. A pesquisa foi realizada entre maio e setembro de 2019 e foi submetida ao Comitê de Ética em Pesquisas do Centro de Ciências da Saúde da Universidade Federal da Paraíba e aprovada sob o Parecer $n^{\circ} 3.430 .175, \mathrm{n}^{\circ}$ CAAE 15399019.4.0000.5188. Fizeram parte da pesquisa os discentes maiores de 18 anos regularmente matriculados no curso de farmácia da UFPB que ingressaram entre no curso entre os anos de 2014 a 2019, e que assinaram o Termo de Consentimento Livre Esclarecido (TCLE) disponibilizado pelo pesquisador. Foram excluídos os discentes que confirmaram história prévia de ansiedade e/ou depressão e os discentes que afirmaram o uso de substâncias psicoativas. Foi realizado um levantamento, a partir de listagem obtida através do Sistema Integrado de Gestão de Atividades Acadêmicas (SIGAA), que consolidou a quantidade de alunos ativos no curso de farmácia da UFPB, matriculados entre 2014 e 2019. Foi identificado um universo total de 342 alunos e realizado um cálculo para populações finitas para seleção da amostragem. A amostra obtida, considerada significativa foi de 182 alunos.

Adicionalmente, os estudantes foram divididos de acordo com o período do curso que estavam cursando. Assim, formando 10 grupos distintos. A coleta de dados foi realizada na Universidade Federal da Paraíba no Campus I, no período de julho a agosto de 2019. O instrumento utilizado foi um questionário semiestruturado, incluindo 21 questões do Inventário de Ansiedade de Beck (BAI) e 9 questões do Patient Health Questionnaire-9 (PHQ-9). 
Os acadêmicos foram selecionados de maneira aleatória e participaram voluntariamente, após serem informados sobre os objetivos da pesquisa e seus aspectos éticos. O questionário foi auto-aplicado, com preenchimento individual e ocorreu no horário de funcionamento do curso (07:00-18:00h), com tempo previamente estabelecido e cedido pelos professores, suficiente paras respostas. Todos os dados levantados nos grupos de estudantes foram duplamente digitados em base de dados e testados quanto à distribuição normal pelo teste de Kolmogorov-Smirnov, antes da seleção dos testes estatísticos. Para comparação dos resultados entre os grupos foi utilizado o teste $t$ de Student. Para análise de correlações entre dados foi utilizado coeficiente de Pearson. A organização da base de dados e a análise estatística foi realizada por meio dos programas Microsoft Excel® 2007 e SPSS ${ }^{2} 20.0$ para Windows.

\section{Resultados}

Foram coletados 245 questionários, $183(74,7 \%)$ preencheram os critérios de inclusão e exclusão previamente definidos. As características demográficas da população estudada estão demonstradas Tabela 1. A maioria dos entrevistados eram solteiros $179(97,8 \%)$, sendo $2(1,1 \%)$ casados e $2(1,1 \%)$ divorciados. Em relação a idade os estudantes apresentavam em média 21,5 anos $(\mathrm{DP}= \pm 3,16)$.

Tabela 1. Caracterização sociodemográfica dos estudantes.

\begin{tabular}{lcc}
\hline Variáveis sociodemográficas & $\mathrm{n}$ & $\%$ \\
\hline Gênero & 118 & 64,5 \\
Feminino & 65 & 35,5 \\
Masculino & & \\
Idade & 98 & 53,6 \\
18 a 21 anos & 69 & 37,6 \\
22 a 25 anos & 11 & 6,0 \\
26 a 29 anos & 2 & 1,1 \\
30 a 33 anos & 2 & 1,1 \\
34 a 37 anos & 1 & 0,5 \\
38 a 41 anos & & 0,5 \\
Renda (em salário mínimo) & 1 & 31,7 \\
Até 1 & 58 & 35,0 \\
1 a 2 & 64 & 22,4 \\
2 a 3 & 41 & 10,4 \\
Acima de 4 & 19 & \\
\hline
\end{tabular}

Fonte: Autores (2021).

Ao levantar os dados relacionados aos scores de classificação de depressão segundo escala do PHQ-9, 32,2\% dos estudantes apresentaram score compatível com depressão mínima e 33,9\% dos discentes com depressão leve. Entretanto, $21,9 \%$ dos alunos demostraram score convergente com depressão moderada, 8,2\% com depressão moderadamente grave e $3,8 \%$ com depressão grave.

Avaliando a distribuição por período dos 62 alunos (34\%), que obtiveram score compatível com depressão de moderada a grave, foi possível observar que a maioria dos alunos, estavam cursando o sexto período (19\%), seguido dos alunos que cursavam o primeiro período (18\%), o segundo (15\%), o terceiro (11\%), o oitavo (11\%), o quarto (7\%), o sétimo (6\%), o nono (5\%), o décimo (5\%) e o quinto período (3\%). 
A Tabela 2 demostra os resultados inerentes à frequência e a porcentagem de cada sintoma relatado pelos estudantes na escala do PHQ-9. Onde a maior frequência de respostas positivas foi para o sintoma "Se sentir cansado/a ou com pouca energia" (89,6\%), seguido do sintoma "Pouco interesse ou pouco prazer em fazer as coisas" (79,2\%) e do sintoma "Se sentir "para baixo", deprimido/a ou sem perspectiva" (73,8\%). Enquanto o sintoma menos frequente foi "Pensar em se ferir de alguma maneira ou que seria melhor estar morto/a".

Tabela 2. Frequência de sintomas depressivos.

\begin{tabular}{lcc}
\hline Sintomas & n & $\%$ \\
\hline Pouco interesse ou pouco prazer em fazer as coisas & 145 & 79,2 \\
Se sentir "para baixo", deprimido/a ou sem perspectiva & 135 & 73,8 \\
$\begin{array}{l}\text { Dificuldade para pegar no sono ou permanecer dormindo, ou dormir mais do que } \\
\text { de costume }\end{array}$ & 111 & 60,7 \\
Se sentir cansado/a ou com pouca energia & 164 & 89,6 \\
Falta de apetite ou comendo demais & 105 & 57,4 \\
$\begin{array}{l}\text { Se sentir mal consigo mesmo/a - ou achar que você é um fracasso ou que } \\
\text { decepcionou sua família ou você mesmo/a }\end{array}$ & 89 & 48,6 \\
$\begin{array}{l}\text { Dificuldade para se concentrar nas coisas, como ler o jornal ou ver televisão } \\
\text { Lentidão para se movimentar ou falar, a ponto das outras pessoas perceberem? Ou } \\
\text { o oposto - estar tão agitado/a ou irrequieto/a que você fica andando de um lado }\end{array}$ & 58 & 51,4 \\
para o outro muito mais do que de costume & & 31,7 \\
Pensar em se ferir de alguma maneira ou que seria melhor estar morto/a & & 19 \\
\hline
\end{tabular}

Fonte: Autores (2021).

Ao avaliar a estratificação dos níveis de ansiedade, de acordo com a escala de BAI, 56,4\% dos discentes obtiveram score coincidente com ansiedade mínima, 27,7\% com ansiedade leve. No entanto, $12 \%$ dos alunos atingiram score consonante com ansiedade moderada, seguido $6 \%$ com score compatível com ansiedade grave.

$\mathrm{Na}$ avalição dos dados, observou-se que maioria dos alunos que obtiveram scores compatível com ansiedade moderada a grave, maioria era do primeiro período (25\%), seguido do oitavo período (21\%), quarto (18\%), nono (9\%), sexto (9\%) segundo (6\%), terceiro $(6 \%)$, quinto $(3 \%)$, sétimo $(3 \%)$.

Adicionalmente, a Tabela 3 apresenta a frequência e a porcentagem dos sintomas ansiosos relatados pelos entrevistados na escala BAI. Assim, o sintoma mais frequente relatado entre os estudantes foi "Nervoso" (77,6\%), o segundo sintoma mais relatado foi "Incapaz de relaxar" (67,2\%), seguido do sintoma "Medo que aconteça o pior" (55,7\%). Entretanto, o sintoma "Sensação de desmaio" foi o menos relatado ente os estudantes (8,9\%), seguido de "Rosto afogueado" (12\%). 
Tabela 3. Frequência de sintomas ansiosos.

\begin{tabular}{|c|c|c|}
\hline Sintomas & $\mathrm{N}$ & $\%$ \\
\hline Dormência ou formigamento & 54 & 29,5 \\
\hline Sensação de calor & 80 & 47,7 \\
\hline Tremores nas pernas & 48 & 26,5 \\
\hline Incapaz de relaxar & 123 & 67,2 \\
\hline Medo que aconteça o pior & 102 & 55,7 \\
\hline Atordoado ou tonto & 69 & 37,7 \\
\hline Palpitação ou aceleração do coração & 92 & 50,3 \\
\hline Sem equilíbrio & 46 & 25,2 \\
\hline Aterrorizado & 55 & 30,1 \\
\hline Nervoso & 142 & 77,6 \\
\hline Sensação de sufocação & 54 & 29,5 \\
\hline Tremores nas mãos & 54 & 29,5 \\
\hline Trêmulo & 48 & 26,6 \\
\hline Medo de perder o controle & 83 & 43,7 \\
\hline Dificuldade de respirar & 57 & 31,1 \\
\hline Medo de morrer & 44 & 24 \\
\hline Assustado & 73 & 39,9 \\
\hline Indigestão ou desconforto no abdômen & 77 & 42,1 \\
\hline Sensação de desmaio & 18 & 9,8 \\
\hline Rosto afogueado & 22 & 12 \\
\hline Suor (não devido ao calor) & 42 & 23 \\
\hline
\end{tabular}

Fonte: Autores (2021).

Adicionalmente, foi realizada a correlação de Pearson (Tabela 4) entre Questionário de Saúde do Paciente-9, o Inventário Beck de ansiedade, a idade e a renda dos estudantes. Os resultados demostraram correlação positiva ( $\mathrm{r}=0,672$ ) entre os escores compatíveis com depressão e os scores de sintomatologia ansiosa. Ademais, utilizando a correlação de Pearson observou-se uma correlação negativa $(r=-0,216)$ entre a idade e os scores da sintomatologia do BAI. Isso não foi observado, quando a idade foi correlacionada com aos scores dos sintomas depressivos $(r=0,059)$. Além disso, não houve correlação entre ao score da escala do PHQ-9 $(r=0,003)$ nem do BAI $(r=0,033)$ com a renda dos estudantes. 
Tabela 4. Correlação das variáveis com os scores das escalas.

\begin{tabular}{lccc}
\hline & Score PHQ-9 & Idade & Renda \\
\hline Score PHQ-9 & $0,672^{*}$ & 0,059 & 0,003 \\
Score BAI & $0,672^{*}$ & $-0,216^{* *}$ & 0,033 \\
\hline
\end{tabular}

Legenda: ** A correlação é significativa no nível 0,01. * A correlação é significativa no nível 0,05. Fonte: Autores (2021).

\section{Discussão}

O presente trabalho evidencia a importância do rastreamento em saúde nos transtornos mentais, que apresentam crescimento exponencial nos últimos anos. Embora os sintomas de depressão sejam predominantes entre os pacientes da atenção primária, poucos pacientes discutem esses sintomas diretamente com seus médicos. Outro fato importante, é que apenas dois terços dos pacientes da atenção primária com depressão apresentam sintomas somáticos (por exemplo, dor de cabeça, problemas nas costas ou dor crônica), dificultando assim sua detecção (Ansseau et al., 2004).

Evidências trazem que a depressão é uma condição frequentemente não detectada na ausência de rastreamento, um estudo randomizado que avaliou o efeito do rastreamento para depressão, observou o aumento do diagnóstico na atenção primária (Pignone, M. P.; Gaynes, B. N.; Rushton, J. L.; Burchell, C. M, Orleans, C. T.; Mulrow, C. D.; Lohr, 2002). Em outro estudo, o rastreamento do paciente atrelado ao envolvimento da equipe clínica permitiu uma melhor reposta a depressão e a remissão (O’Connor, 2009). Um rastreamento adequado, permite uma intervenção precoce e um desfecho mais promissor para o paciente.

Diversas revisões sistemáticas abordam os efeitos do rastreamento da depressão para uma ampla gama de resultados clinicamente relevantes (Gilbody et al., 2003; Pignone, M. P.; Gaynes, B. N.; Rushton, J. L.; Burchell, C. M, Orleans, C. T.; Mulrow, C. D.; Lohr, 2002). Uma meta-análise subsequente de 16 estudos com 7576 pacientes descobriu que o uso de instrumentos de rastreamento de depressão ou de busca de casos na atenção primária ou em outros ambientes de saúde foi associado a um aumento modesto no reconhecimento da depressão pelos médicos (risco relativo [RR] 1,27, IC 95\% 1,02-1,59) e um impacto não significativo no início de qualquer tratamento (RR 1,30; IC 0,97-1,76), sem influência na prescrição de antidepressivos (RR 1,20, IC 0,87-1,66)(Gilbody et al., 2003).

Nesse sentido foi realizado um rastreamento em saúde para ansiedade e depressão nos discentes de farmácia da Universidade Federal da Paraíba, a fim de avaliar a incidência desses transtornos mentais. É sabido que os dados sobre as doenças mentais no contexto universitário são expressivos. Uma revisão sistemática de 2016 que analisou 77 estudos com um total de 62.728 estudantes de medicina incluídos, realizada pela National University of Singapore observou a prevalência global de $28 \%$ de depressão (95\% [IC] 24,2-32,1\%) em estudantes de medicina (Puthran et al., 2016).

Nossa análise apontou que 33,9\% da amostra apresentaram scores compatíveis com depressão moderada a grave, e $18 \%$ apresentaram scores compatíveis com ansiedade moderada a grave. Esses dados corroboram com a literatura, uma revisão sistemática de 2020, que avaliou a prevalência de depressão em estudantes universitários de países de baixa e média renda, analisou 37 estudos contemplando cerca de 75.000 indivíduos em 20 países detectou uma prevalência de 24,4\% (IC 95\%, 19,2\% a 30,5\%) de sintomas depressivos (Akhtar et al., 2020).

Outra revisão sistemática de 2012 que avaliou a prevalência de depressão em estudantes universitários em geral observou que. As taxas de prevalência relatadas variaram de $10 \%$ a $85 \%$ com uma prevalência média ponderada de $30,6 \%$ (Ibrahim et al., 2013). Embora esses resultados sejam inferiores aos nossos achados, isso pode ser explicado tanto pelas 
diferenças na população avaliada, visto que nosso estudo foi conduzido no curso de farmácia, um curso com elevada complexidade e carga horária, bem como diferenças nas escalas aplicadas.

Assim como outros instrumentos de triagem, o PHQ-9 não é o suficiente para fechar um diagnóstico definitivo de transtorno depressivo, necessitando de uma avaliação médica diagnóstica, que seja bem criteriosa. Vale ressaltar, que o questionário utilizado nesse estudo é apontado como um dos mais adequado para detecção e monitoramento da depressão nos mais diversos tipos populações, possuindo sensibilidade de 88\% e especificidade 85\% (Huang et al., 2006; Levis et al., 2019). Segundo, Miyazaki e Silvares (1997) as altas taxas de transtornos depressivos são mais comuns no início do curso em função da fase estressante fase de adaptação. Então, levantamos o período da graduação daqueles estudantes que apresentaram uma pontuação igual ou maior que $10(n=62)$. Entretanto, nesse trabalho, a maioria dos alunos que obtiveram scores compatíveis com depressão moderada a grave cursavam o sexto período. Sugerindo assim, que o sexto período seja estressante, visto que este período apresenta a segunda maior carga horária de disciplinas obrigatórias. A segunda maior taxa foi encontrada no primeiro período, seguido do segundo. O que venha a ser justificada pela fase de adaptação ao novo estilo de vida, mudança de cidade e afastamento da família que são fatores desencadeantes de estresse e, que é intimamente relacionado ao desenvolvimento de transtornos depressivos.

A escala utilizada no presente trabalho abrange alguns sintomas elencados no DMS-V (2014), no qual as frequências dos sintomas nos estudantes estão apresentadas na tabela 3. O DMS-V, estabelece alguns sintomas que são considerados critérios de diagnósticos para depressão maior e que devem persistir por duas semanas. Esse manual preconiza que "humor deprimido" ou "perda de interesse ou prazer" devem aparecer nos critérios de diagnóstico, adicionados a outros sintomas descritos no manual. Curiosamente, esses sintomas estão presentes entre os três mais relatados pelos discentes, sugerindo assim uma investigação mais detalhada para descartar ou confirmar a ocorrência de transtornos depressivos. Sabe-se que a universidade é ambiente altamente competitivo e cheio de insatisfações o que é considera um fator para sintomas ansiosos (Amr, 2008).

Para avaliar a prevalência de transtorno ansioso nos universitários utilizamos o BAI, que é considerado uma escala adequada para rastrear e mensurar a estratificação da ansiedade por oferecer uma alta confiabilidade e validade satisfatória (Liang et al., 2018). A escala utilizada apresenta alta sensibilidade, assim, $78 \%$ dos alunos apresentaram scores abaixo de 20 pontos. No entanto, que configura um fraco indício para o desenvolvimento transtornos ansiosos. Curiosamente, os resultados indicaram uma elevada taxa de ansiedade moderada a grave de $18 \%$ dos alunos. Não foram encontrados dados na literatura que mesurem os transtornos ansiosos em estudantes de farmácia. Porém, dados semelhantes foram encontrados em um estudo realizado com 234 discentes da Faculdade de Medicina Pernambuco (Vasconcelos et al., 2015). Vale ressaltar que curso de farmácia apresenta uma elevada e extenuante carga horária, com uma grade curricular com disciplinas extremante complexas e com atividades extracurriculares. Com o intuito de cumprir todos os prazos, os discentes estão em constante privação de sono, que segundo o estudo Almondes e Araújo (2003), utilizando a escala IDATE em estudantes da área da saúde, privação de sono em alunos é considerado um "gatilho" para ansiedade moderada a grave.

Ao avaliar a prevalência da sintomatologia ansiosa nos alunos de farmácia, quando a distribuição foi realizada por período, dos 33 alunos que obtiveram scores $\geq 20$, a maioria encontrava cursando o primeiro período de farmácia. Segundo o estudo realizado por Mathew et al. (2019), que avaliou os fatores estressores em alunos de farmácia, no qual o primeiro período seria o mais estressante, visto que a cobrança para a conquista de um diploma em bacharel, muitas expectativas em relação ao futuro, poderia ser uma consequência e estar relacionado com sintomas ansiosos. Adicionalmente, alunos do oitavo período apresentaram a segunda maior prevalência, no qual a pontuação foi coincidente com ansiedade moderada a grave, não existindo dados na literatura que justifiquem esse resultado. Sugerimos então que tal dado está relacionado à inserção das cadeiras voltadas a análises clínica e preocupação com o desempenho em uma nova área do curso. 
Quando avaliados os sintomas mais relatados no BAI, o mais frequente foi "Nervoso", seguido de "Incapaz de relaxar" e "Medo que aconteça o pior". O que resulta em uma situação de vulnerabilidade ao sofrimento psicológico dos alunos já apontado em outros estudos (Baldassin et al., 2006; Carlotto et al., 2006).

Entretanto, na literatura foi encontrado um estudo semelhante que tinha a finalidade de relatar os sintomas ansiosos mais comuns em universitários. Ademais, em um estudo com 1.046 estudantes que se preparavam para o vestibular, utilizando o mesmo instrumento, os três sintomas mais frequente entre os alunos foram os mesmos que foram relatados em nosso estudo. Esses sintomas acabam sendo bastante frequentes em TAG (Rodrigues \& Pelisoli, 2008). No entanto, para finalizar o diagnóstico faz se necessário uma avaliação médica com esses estudantes.

A fim de mensurar o nível de relação entre o desenvolvimento da depressão atrelado a ansiedade, como também avaliar os scores finais das escalas PHQ-9 e BAI, com a idade e renda utilizou-se a correlação de Pearson (Spiegel, 1993; Toledo, G. L.; Ovalle, 1985). Segundo dados da literatura, os valores de correlação podem ser classificados como fraco moderado ou forte, onde $r=0,10$ até 0,39 demonstra uma correlação fraca; $r=0,40$ até 0,69 aponta uma correlação moderada e $r=0,70$ até 1 evidencia uma correlação forte. Essa correlação de Pearson pode ser positiva ou negativa, quando venha a ser positiva as duas variáveis se movem na mesma direção, e quando negativa, as duas variáveis seguem direções opostas (Dancey, C. P.; Reidy, 2006).

$\mathrm{Na}$ análise do desenvolvimento da ansiedade concomitantemente a depressão, observou se uma correlação positiva moderada (tabela 5), demostrando assim que quanto mais os estudantes apresentaram indícios ansiosos, maiores foram seus scores para sintomatologia depressiva. Assim esse resultado se encontra em consonância com a literatura, visto que e em quase dois terços dos casos de transtornos ansioso existe uma a co-ocorrencia com outras comorbidades mentais, no qual a mais comum é a depressão (Wittchen \& Jacobi, 2005).

Mais da metade dos discentes que participaram desse estudo tinham idade entre 18 e 21 anos. Ao estimar a correlação entre os transtornos depressivos e a idade dos estudantes não houve correlação significativa com os scores obtidos no PHQ-9. Esse resultado está consonância com estudo Bayrame e Bilgel (2008), onde a variável idade pareceu não está correlacionada com os scores finais da escala de depressão. No entanto, houve uma relação negativa fraca entre idade dos alunos e os scores finais obtidos para os transtornos ansiosos. Isso significa que quanto menor a idade dos alunos, a sintomatologia ansiosa é mais frequente, favorecendo assim o desencadeamento de transtornos ansiosos. Kessler et al. (2005), relata que os transtornos ansiosos em sua maioria começam precocemente.

Ao avaliar o desenvolvimento dos transtornos ansiosos e depressivos nos discentes de farmácia em relação renda familiar não foi observada correlação significativa. Bayrame e Bilgel (2008), realizado um estudo com estudantes turcos, onde foi observado que os maiores scores na escala depressão foi daqueles estudantes eram referentes àqueles que apresentavam as menores rendas. Entretanto, não encontrado nenhuma relação da sintomatologia ansiosa com a renda familiar. Sugerindo assim, que os problemas acadêmicos nos estudantes de Farmácia da UFPB refletem mais no desenvolvimento de transtornos mentais que os problemas relacionados ao âmbito da vida pessoal deles.

\section{Conclusão}

Com o presente trabalho foi possível concluir que os acadêmicos de farmácia de uma Universidade Pública do Brasil apresentam uma forte tendência ao desenvolvimento de transtornos depressivos e ansiosos. Os sintomas mais frequentes como, pouco interesse ou pouco prazer em fazer as atividades, o sentimento de "para baixo", deprimido/a ou sem perspectiva, e o sentimento de cansado/a ou com pouca energia no caso da depressão e os sintomas de incapacidade de relaxar, temor que aconteça o pior e o nervosismo para a ansiedade foram associados diretamente a redução da qualidade de vida dos alunos, por acarretar algum tipo de sofrimento aos mesmos. Também foi possível observar correlação entre escores compatíveis com 
ansiedade e depressão na amostra estudada. Ademais, na amostra estudada notou-se que quanto menor a idade, mais frequente a presença de sintomas ansiosos. Além disso, a renda pareceu não influenciar na presença dos transtornos, indicando assim que os fatores acadêmicos são considerados fator de risco mais relevantes para tais transtornos. O presente trabalho, ao passo que demonstra uma realidade alarmante, abre espaço para investigações posteriores, multicêntricas, bem como, voltadas a identificação dos fatores de risco ligados ao adoecimento mental de estudantes da área da saúde.

\section{Agradecimentos}

Este trabalho contou com apoio financeiro da Chamada $\mathrm{n}^{\circ} \quad 03 / 2020$ Produtividade em Pesquisa PROPESQ/PRPG/UFPB código do projeto de pesquisa no SIGAA PVG13392-2020.

\section{Referências}

Adewuya, A. O., Ola, B. A., Aloba, O. O., Mapayi, B. M., \& Oginni, O. O. (2006). Depression amongst Nigerian university students: Prevalence and sociodemographic correlates. Social Psychiatry and Psychiatric Epidemiology, 41(8), 674-678. https://doi.org/10.1007/s00127-006-0068-9

Akhtar, P., Ma, L., Waqas, A., Naveed, S., Li, Y., Rahman, A., \& Wang, Y. (2020). Prevalence of depression among university students in low and middle income countries (LMICs): a systematic review and meta-analysis. Journal of Affective Disorders, 274(November 2019), 911-919. https://doi.org/10.1016/j.jad.2020.03.183

Amr, M. (2008). Does Gender Predict Medical Students' Stress in Mansoura, Egypt? Medical Education Online, 13, 1-9. https://doi.org/10.3885/meo.2008.res00273

Ansseau, M., Dierick, M., Buntinkx, F., Cnockaert, P., De Smedt, J., Van Den Haute, M., \& Vander Mijnsbrugge, D. (2004). High prevalence of mental disorders in primary care. Journal of Affective Disorders, 78(1), 49-55. https://doi.org/10.1016/S0165-0327(02)00219-7

Baldassin, S., Martins, L. C., \& Guerra De Andrade, A. (2006). Traços de ansiedade entre estudantes de medicina. Arq Med ABC, 31(1), $27-31$.

Carlotto, M. S., Nakamura, A. P., \& Câmara, S. G. (2006). Síndrome de Burnout em estudantes universitários da área da saúde. Psico (Porto Alegre), 57-62.

Cavestro, J. D. M., \& Rocha, F. L. (2006). Prevalência de depressão entre estudantes universitários. Jornal Brasileiro de Psiquiatria, 55(4), 264-267. https://doi.org/10.1590/S0047-20852006000400001

Cerchiari, E. A. N., Caetano, D., \& Faccenda, O. (2005). Prevalência de transtornos mentais menores em estudantes universitários. Estudos de Psicologia (Natal), 10(3), 413-420. https://doi.org/10.1590/s1413-294x2005000300010

Cestari, V. R. F., Barbosa, I. V., Florêncio, R. S., Pessoa, V. L. M. de P., \& Moreira, T. M. M. (2017). Estresse em estudantes de enfermagem: estudo sobre vulnerabilidades sociodemográficas e acadêmicas. Acta Paulista de Enfermagem, 30(2), 190-196. https://doi.org/10.1590/1982-0194201700029

Chao, D. C., \& Chu-Lien, R. (2012). Managing Perceived Stress Among College Students: The Roles of Social Support and. Journal of College Counseling, $15(5), 5-21$.

Christopher, G., \& MacDonald, J. (2005). The impact of clinical depression on working memory. Cognitive Neuropsychiatry, 10(5), 379-399. https://doi.org/10.1080/13546800444000128

Dancey, C. P., Reidy, J. (2006). Análise de correlação: o r de Pearson. In P. A. Artemed (Ed.), Estatística sem matemática para psicologia (pp. 178-216).

Gilbody, S., Whitty, P., Grimshaw, J., \& Thomas, R. (2003). Educational and Organizational Interventions to Improve the Management of Depression in Primary Care: A Systematic Review. Journal of the American Medical Association, 289(23), 3145-3151. https://doi.org/10.1001/jama.289.23.3145

Hasin, D. S., Sarvet, A. L., Meyers, J. L., Saha, T. D., Ruan, W. J., Stohl, M., \& Grant, B. F. (2018). Epidemiology of adult DSM-5 major depressive disorder and its specifiers in the United States. JAMA Psychiatry, 75(4), 336-346. https://doi.org/10.1001/jamapsychiatry.2017.4602

Huang, F. Y., Chung, H., Kroenke, K., Delucchi, K. L., \& Spitzer, R. L. (2006). Using the Patient Health Questionnaire-9 to measure depression among racially and ethnically diverse primary care patients. Journal of General Internal Medicine, 21(6), 547-552. https://doi.org/10.1111/j.1525-1497.2006.00409.X

Ibrahim, A. K., Kelly, S. J., Adams, C. E., \& Glazebrook, C. (2013). A systematic review of studies of depression prevalence in university students. Journal of Psychiatric Research, 47(3), 391-400. https://doi.org/10.1016/j.jpsychires.2012.11.015

Jadhakhan, F., Lindner, O. C., Blakemore, A., \& Guthrie, E. (2019). Prevalence of common mental health disorders in adults who are high or costly users of healthcare services: Protocol for a systematic review and meta-analysis. In BMJ Open (Vol. 9, Issue 9, p. 28295). BMJ Publishing Group. https://doi.org/10.1136/bmjopen-2018-028295

Khushalani, J. S., Qin, J., Cyrus, J., Buchanan Lunsford, N., Rim, S. H., Han, X., Yabroff, K. R., \& Ekwueme, D. U. (2018). Systematic review of healthcare costs related to mental health conditions among cancer survivors. In Expert Review of Pharmacoeconomics and Outcomes Research (Vol. 18, Issue 5, pp. 505-517). Taylor and Francis Ltd. https://doi.org/10.1080/14737167.2018.1485097 
Kroenke, K., Spitzer, R. L., Williams, J. B. W., \& Löwe, B. (2010). The Patient Health Questionnaire Somatic, Anxiety, and Depressive Symptom Scales: A systematic review. General Hospital Psychiatry, 32(4), 345-359. https://doi.org/10.1016/j.genhosppsych.2010.03.006

Levis, B., Benedetti, A., \& Thombs, B. D. (2019). Accuracy of Patient Health Questionnaire-9 (PHQ-9) for screening to detect major depression: Individual participant data meta-analysis. The BMJ, 365. https://doi.org/10.1136/bmj.11476

Liang, Y., Wang, L., \& Zhu, J. (2018). Factor structure and psychometric properties of Chinese version of Beck Anxiety Inventory in Chinese doctors. Journal of Health Psychology, 23(5), 657-666. https://doi.org/10.1177/1359105316658971

Luppa, M., Heinrich, S., Angermeyer, M. C., König, H. H., \& Riedel-Heller, S. G. (2007). Cost-of-illness studies of depression. A systematic review. In Journal of Affective Disorders (Vol. 98, Issues 1-2, pp. 29-43). https://doi.org/10.1016/j.jad.2006.07.017

M.S., H., M.G., H., \& D.G., S. (1991). Dimensions of alexithymia and their relationships to anxiety and depression. Journal of Personality Assessment, 56(2), 227-237.

http://www.embase.com/search/results?subaction=viewrecord\&from=export\&id=L21839377\%5Cnhttp://sfx.library.uu.nl/utrecht?sid=EMBASE\&issn=00223 891\&id=doi:\&atitle=Dimensions+of+alexithymia+and+their+relationships+to+anxiety+and+depression.\&stitle=J+Pers+Asses

Magán, I., Sanz, J., \& García-Vera, M. P. (2008). Psychometric properties of a Spanish version of the Beck anxiety inventory (BAI) in general population. Spanish Journal of Psychology, 11(2), 626-640. https://doi.org/10.1017/s1138741600004637

McConville, J., McAleer, R., \& Hahne, A. (2017). Mindfulness Training for Health Profession Students-The Effect of Mindfulness Training on Psychological Well-Being, Learning and Clinical Performance of Health Professional Students: A Systematic Review of Randomized and Non-randomized Controlled Trials. Explore: The Journal of Science and Healing, 13(1), 26-45. https://doi.org/10.1016/j.explore.2016.10.002

O’Connor, E. A. (2009). Clinical Guidelines Screening for Depression in Adult Patients in Primary Care Settings : Annals of Internal Medicine.

Oliveira, R. E. C, Morais, A. (2015). Vivências acadêmicas e adaptação de estudantes de uma universidade pública federal do Estado do Paraná. 547-568.

Osman, A., Hoffman, J., Barrios, F. X., Kopper, B. A., Breitenstein, J. L., \& Hahn, S. K. (2002). Factor structure, reliability, and validity of the beck anxiety inventory in adolescent psychiatric inpatients. Journal of Clinical Psychology, 58(4), 443-456. https://doi.org/10.1002/jclp.1154

Pignone, M. P., Gaynes, B. N.,Rushton, J. L., Burchell, C. M, Orleans, C. T., Mulrow, C. D.; \& Lohr, K. N. (2002). Screening for depression in adults: a summary of the evidence for the US Preventive Services Task Force. Annals of Internal Medicine, v. 136, n. 10, p. 765-766, 2002., 136(n 10), p 765-766. https://doi.org/10.1191/0269216303pm756ra

Pillay, N., Ramlall, S., \& Burns, J. K. (2016). Spirituality, depression and quality of life in medical students in KwaZulu-Natal. South African Journal of Psychiatry, 22(1), 1-6. https://doi.org/10.4102/sajpsychiatry.v22i1.731

Puthran, R., Zhang, M. W. B., Tam, W. W., \& Ho, R. C. (2016). Prevalence of depression amongst medical students: A meta-analysis. Medical Education, 50(4), 456-468. https://doi.org/10.1111/medu.12962

Rodrigues, D. G., \& Pelisoli, C. (2008). Ansiedade em vestibulandos: Um estudo exploratório. Revista de Psiquiatria Clinica, 35(5), 171-177. https://doi.org/10.1590/S0101-60832008000500001

Spiegel, M. R. (1993). Correlação de Pearson. Estatística (Pearson Makron Books (ed.); 3 ed).

Thurber, C. A., Walton, E., Murray, R. D., Frankowski, B. L., Gereige, R. S., Mears, C. J., Roland, M. M., Young, T. L., Grant, L. M., Hyman, D., Magalnick, H., Monteverdi, G. J., Pattishall, E. G., LaCursia, N., Mazyck, D., Vernon-Smiley, M., Wallace, R., \& Li, S. (2007). Preventing and treating homesickness. Pediatrics, 119(1), 192-201. https://doi.org/10.1542/peds.2006-2781

Toledo, G. L., Ovalle, I. I. (1985). Estatística básica (Atlas (ed.); 2 ed).

Vasconcelos, T. C. de, Dias, B. R. T., Andrade, L. R., Melo, G. F., Barbosa, L., \& Souza, E. (2015). Prevalência de Sintomas de Ansiedade e Depressão em Estudantes de Medicina TT - Prevalence of Anxiety and Depression Symptoms among Medicine Students. Revista Brasileira de Educação Médica, 39(1), 135-142. https://doi.org/10.1590/1981-52712015v39n1e00042014

Vázquez Morejón, A. J., Vázquez-Morejón Jiménez, R., \& Zanin, G. B. (2014). Beck anxiety inventory: Psychometric characteristics in a sample from the clinical spanish population. Spanish Journal of Psychology, 17(2), 1-11. https://doi.org/10.1017/sjp.2014.76

Wittchen, H. U., \& Jacobi, F. (2005). Size and burden of mental disorders in Europe - A critical review and appraisal of 27 studies. European Neuropsychopharmacology, 15(4), 357-376. https://doi.org/10.1016/j.euroneuro.2005.04.012 